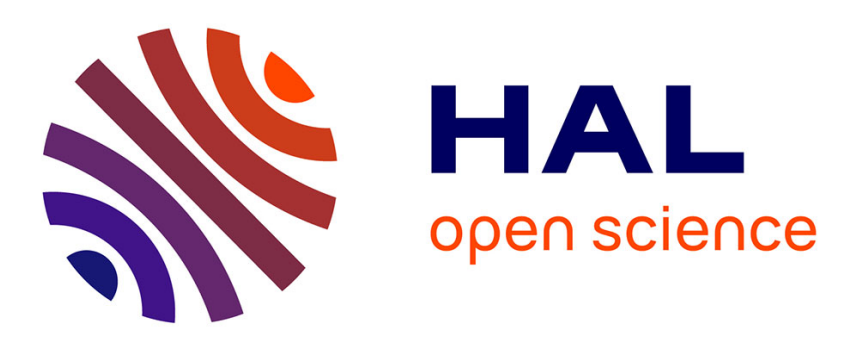

\title{
Power Allocation for Minimizing Energy Consumption of OFDMA Downlink with Cell DTx
}

\author{
Rémi Bonnefoi, Christophe Moy, Haïfa Farès, Jacques Palicot
}

\section{To cite this version:}

Rémi Bonnefoi, Christophe Moy, Haïfa Farès, Jacques Palicot. Power Allocation for Minimizing Energy Consumption of OFDMA Downlink with Cell DTx. 24th International Conference on Telecommunications (ICT 2017), May 2017, Limassol, Cyprus. 10.1109/ICT.2017.7998232 . hal-01504248v8

\section{HAL Id: hal-01504248 \\ https://hal.science/hal-01504248v8}

Submitted on 27 Aug 2018

HAL is a multi-disciplinary open access archive for the deposit and dissemination of scientific research documents, whether they are published or not. The documents may come from teaching and research institutions in France or abroad, or from public or private research centers.
L'archive ouverte pluridisciplinaire HAL, est destinée au dépôt et à la diffusion de documents scientifiques de niveau recherche, publiés ou non, émanant des établissements d'enseignement et de recherche français ou étrangers, des laboratoires publics ou privés. 


\title{
Power Allocation for Minimizing Energy Consumption of OFDMA Downlink with Cell DTx
}

\author{
Rémi Bonnefoi*, Christophe Moy*, Haïfa Farès ${ }^{* \dagger}$ and Jacques Palicot* \\ *CentraleSupélec/IETR, CentraleSupélec Campus de Rennes, 35510 Cesson-Sévigné, France \\ Email:\{remi.bonnefoi, christophe.moy, haifa.fares, jacques.palicot\}@centralesupelec.fr \\ ${ }^{\dagger}$ CEA Saclay, SPEC, URA 2464, F-91191 Gif-Sur-Yvette, France
}

\begin{abstract}
With Cell DTx, a base station can dynamically be switched to sleep mode during very short periods (inferior to frame duration) in order to reduce its energy consumption. In this paper, we study the problem of energy consumption minimization with Cell DTx for the downlink of an Orthogonal Frequency Division Multiple Access (OFDMA) base station. We show that this problem can be rewritten as a convex problem. Then, we derive interpretable expressions for the transmit power and sleep mode duration which minimize the energy consumption. Besides, we show that resource allocation algorithms already proposed for transmit power minimization have near optimal performance. Simulation results show that up to $38 \%$ of base station energy consumption can be saved with Cell DTx.
\end{abstract}

\section{INTRODUCTION}

Nowadays, base station use around $40-50 \%$ of the total energy consumed by wireless mobile networks [1]. Therefore, an improvement of base stations energy efficiency will greatly reduce the carbon footprint of cellular networks.

Cell DTx has recently been proposed for reducing the base energy consumption [2]. With this solution, a base station can be switched to sleep mode during very short periods (a few milliseconds) to save energy. During these short sleeps, some of the elements of the RF chain such as the power amplifier are switched off. However, for a given throughput (or capacity) with Cell DTx, an increase in sleep mode duration increases the instantaneous transmit power and vice versa. Consequently, there is a trade-off between the dynamic energy consumption, which depends on the transmit power, and the static power consumption, which is reduced by increasing sleep mode duration. Finding the power allocation which minimizes the base station energy consumption aims at finding the trade-off between dynamic and static energy consumption.

The problem of optimal power allocation with Cell DTx was first set in Time Division Multiple Access (TDMA) in [3]. The closed form expression of the optimal power allocation in TDMA has been proposed in [4]. This problem was further investigated in [5] for femtocells and in [6] for macrocells. In [7], authors use the optimal power allocation in TDMA to propose an efficient power allocation in Orthogonal Frequency Division Multiple Access (OFDMA). However, the optimal power allocation has not been studied in this paper. In [8], authors prove that the problem of optimal power allocation with Cell DTx is quasi-convex and show that a water-filling algorithm can be used to find the optimal power allocation. However, the authors did not provide interpretable expressions of the transmit power.

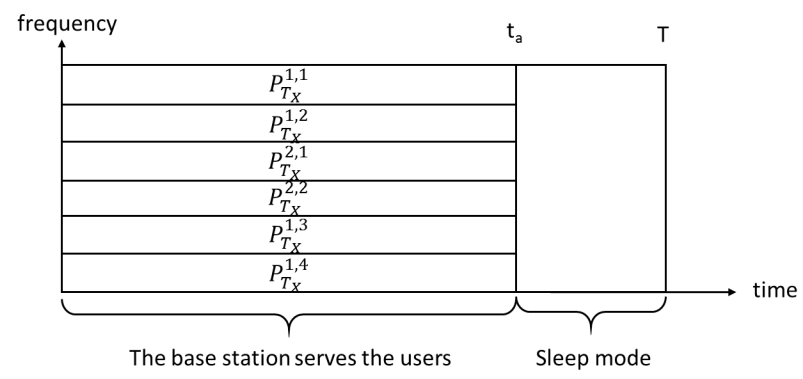

Fig. 1. Frame composed of 6 subchannels each of bandwidth $B_{c}$, two users are served by the base station. For each user and in each subchannel, the power is allocated in order to minimize the base station energy consumption.

In this paper, we first focus on the problem of power allocation and we show that this problem can be rewritten as a convex optimization problem with a variable change. Then we solve this problem, and we show that the optimal power allocation can be computed using only a water-filling algorithm and single variable root-finding algorithms. We finally investigate the problem of resource allocation and we show that resource allocation strategies already proposed for power control [9] (i.e. for minimizing the RF transmit power) give a near optimal performance with Cell DTx.

The rest of this paper is organized as follows. In section II, we introduce the system model and the problem of energy consumption minimization. The problem of power allocation is rewritten in a convex form in section III and solved in section IV. The problem of resource allocation is studied in section V. Some numerical results are provided in section VI and section VII concludes this paper.

\section{System Model And Problem Formulation}

We suppose a base station which serves $N_{u}$ users in $N_{c}$ subchannels of bandwidth $B_{c}$ using OFDMA. The base station allocates each subchannel to a user and adjusts the transmit power in order to reduce its energy consumption. We denote $S_{k}$ the set of subchannels used for user $k$, and $P_{T_{X}}^{k, n}$ the transmit power allocated to user $k$ in channel $n$. As illustrated in figure 1 , the time is divided into frames of duration $T$. In each frame, the base station is active and serves the users during $t_{a}$ and then switches to sleep mode until the end of the frame. We denote $\mu_{a}=\frac{t_{a}}{T}$ the proportion of time used to serve the users. Moreover, the base station provides each user $k \in \llbracket 1, N_{u} \rrbracket$ a given capacity $C_{k}$ :

$$
C_{k}=B_{c} \mu_{a} \sum_{n=1}^{N_{k}} \log _{2}\left(1+\frac{G_{k, n} P_{T_{X}}^{k, n}}{B_{c}}\right),
$$


In this equation, $N_{k}=\left|S_{k}\right|$ denotes the cardinal of $S_{k}$, and $G_{k, n}=\frac{\left|h_{k, n}\right|^{2}}{N_{0}}$ is the channel gain of user $k$ in subchannel $n$ (attenuation, antenna gain, thermal noise, noise figure). Moreover, we use a linear model for the instantaneous base station power consumption [10]:

$$
\left\{\begin{array}{cccc}
P_{\text {supply }} & = & P_{0}+m P_{T_{X}} & \text { when active } \\
P_{\text {supply }} & = & P_{S} & \text { during sleep mode }
\end{array}\right.
$$

Where $P_{0}$ is the static power consumption when the base station is active (processing, cooling, etc.). $P_{S}$ is the base station power consumption during sleep mode, $m$ is the coefficient of the load dependence. $\left.\left.P_{T_{X}} \in\right] 0 ; P_{\text {max }}\right]$ is the total instantaneous RF transmit power which is the sum of the transmit powers allocated in subchannels. $P_{\text {max }}$ denotes the maximum RF transmit power of the base station. In [10] authors list some realistic values for $P_{0}, P_{s}, P_{\text {max }}$, and $m$. In table I, we mention the characteristics used in this paper for numerical evaluations.

TABLE I

POWER VALUES FOR DIFFERENT BASE STATIONS TYPES FROM [10]

\begin{tabular}{|c|c|c|c|c|}
\hline BS type & $P_{\max }(\mathrm{W})$ & $P_{0}(\mathrm{~W})$ & $m$ & $P_{\boldsymbol{s}}(\mathrm{W})$ \\
\hline Macro & 20.0 & 130.0 & 4.7 & 75.0 \\
\hline Femto & 0.05 & 4.8 & 8.0 & 2.9 \\
\hline
\end{tabular}

We now set the problem of energy consumption minimization. In a first step, we only consider the problem of power allocation. We suppose that the sets $S_{k}, \forall k \in \llbracket 1 ; N_{u} \rrbracket$ have been allocated. To minimize the base station energy consumption, we minimize the average power consumption during a frame whose expression can be deduced from (2).

$$
\begin{aligned}
\min _{\mu_{a}, P_{T_{X}}} & \left(1-\mu_{a}\right) P_{s}+\left(P_{0}+m \sum_{k=1}^{N_{u}} \sum_{n=1}^{N_{k}} P_{T_{X}}^{k, n}\right) \mu_{a} \\
\text { S.t. } \quad & \mu_{a} \in[0 ; 1] \\
& C_{k}-B_{c} \mu_{a} \sum_{n=1}^{N_{k}} \log _{2}\left(1+\frac{G_{k, n} P_{T_{X}}^{k, n}}{B_{c}}\right)=0, \forall k \\
& \sum_{k=1}^{N_{u}} \sum_{n=1}^{N_{k}} P_{T_{X}}^{k, n} \leq P_{\max } \\
& P_{T_{X}, n}^{k, n} \geq, \quad \forall k, n
\end{aligned}
$$

\section{Problem ReFormulation}

As proven in appendix $A$, the problem introduced in equation (3) is not convex in that form. In order to rewrite the problem of energy consumption minimization as a convex problem, we first proceed a variable change and we introduce the capacities per subchannel $C_{k, n}$ :

$$
C_{k, n}=B_{c} \mu_{a} \log _{2}\left(1+\frac{G_{k, n} P_{T_{X}}^{k, n}}{B_{c}}\right) .
$$

With these new variables, the objective function of equation (3a) becomes:

$$
\left(1-\mu_{a}\right) P_{s}+\left(P_{0}+m \sum_{k=1}^{N_{u}} \sum_{n=1}^{N_{k}} \frac{B_{c}}{G_{k, n}}\left[2^{\frac{C_{k, n}}{B_{c} \mu_{a}}}-1\right]\right) \mu_{a}
$$

Proposition 1. The objective function introduced in equation (5) is convex.

Proof. $\mu_{a} \mapsto \mu_{a}\left(P_{0}-P_{s}\right)$ is linear. Moreover, the Hessian matrix of the functions $g_{k, n}:\left(\mu_{a}, C_{k, n}\right) \mapsto$ $\frac{m B_{c} \mu_{a}}{G_{k, n}}\left(2^{\frac{C_{k, n}}{B_{c} \mu_{a}}}-1\right)$ is equal to:

$$
H_{k, n}=\frac{m \ln (2)^{2}}{G_{k, n} B_{c} \mu_{a}} 2^{\frac{C_{k, n}}{B_{c} \mu_{a}}}\left(\begin{array}{cc}
\frac{C_{k, n}^{2}}{\mu_{a}^{2}} & -\frac{C_{k, n}}{\mu_{a}} \\
-\frac{C_{k, n}}{\mu_{a}} & 1
\end{array}\right)
$$

$$
\lambda_{1}=0 \text { and } \lambda_{2}=\frac{m \ln (2)^{2}}{G_{k, n} B_{c} \mu_{a}}\left(1+\frac{C_{k, n}^{2}}{\mu_{a}^{2}}\right) 2^{\frac{C_{k, n}}{B_{c} \mu_{a}}} \text { are the }
$$

two eigenvalues of $H_{k, n}$. These eigenvalues are positive, and consequently, $g_{k, n}$ are convex functions.

Thus, equation (5) is convex as the sum of convex functions.

Moreover, constraint (3d) becomes:

$$
\sum_{k=1}^{N_{u}} \sum_{n=1}^{N_{k}} \frac{B_{c}}{G_{k, n}}\left[2^{\frac{C_{k, n}}{B_{c} \mu_{a}}}-1\right] \leq P_{\text {max }} .
$$

An analysis of the Hessian matrix of equation (7) shows that this constraint is not convex. We can change it into a convex constraint without changing the minimization problem. Indeed, the optimal power allocation can't be obtained for $\mu_{a}=0$, as a consequence, the constraint of (7) can be rewritten:

$$
\mu_{a} \sum_{k=1}^{N_{u}} \sum_{n=1}^{N_{k}} \frac{B_{c}}{G_{k, n}}\left[2^{\frac{C_{k, n}}{B c \mu a}}-1\right] \leq \mu_{a} P_{\text {max }}
$$

Moreover, this new constraint is convex (the proof is identical to the one done for the objective function). Finally, we obtain a convex problem for the optimal power allocation with Cell DTx:

$$
\min _{\mu_{a}, C_{k, n}}\left(1-\mu_{a}\right) P_{s}+\left(P_{0}+m \sum_{k=1}^{N_{u}} \sum_{n=1}^{N_{k}} \frac{B_{c}}{G_{k, n}}\left[2^{\frac{C_{k, n}}{B_{c} \mu_{a}}}-1\right]\right) \mu_{a}
$$

$$
\begin{aligned}
& \text { S.t. } \mu_{a} \in[0 ; 1] \\
& \qquad \begin{array}{l}
C_{k}-\sum_{n=1}^{N_{k}} C_{k, n}=0, \forall k \\
\mu_{a} \sum_{k=1}^{N_{u}} \sum_{n=1}^{N_{k}} \frac{B_{c}}{G_{k, n}}\left[2^{\frac{C_{k, n}}{B_{c} \mu_{a}}}-1\right] \leq \mu_{a} P_{\max } \\
C_{k, n} \geq 0, \quad \forall k, n
\end{array}
\end{aligned}
$$

The Karush-Kuhn and Tucker (KKT) conditions can finally be used to solve this problem.

\section{Optimal Power Allocation}

We denote $\mu_{\text {opt }}$ the optimal value of $\mu_{a}$. We calculate the optimal capacity per subchannel using the derivative of the Lagrangian of the studied problem:

$$
C_{k, n}=\left(\frac{C_{k}}{N_{k}^{\prime}}+B_{c} \mu_{o p t} \log _{2}\left(\frac{G_{k, n}}{\left(\prod_{n=1}^{N_{k}^{\prime}} G_{k, n}\right)^{\frac{1}{N_{k}^{\prime}}}}\right)\right)^{\dagger},
$$


where $N_{k}^{\prime}$ denotes the number of subchannels used for user $k$ in which the capacity is non-zero and $a^{\dagger}=\max (a, 0)$.

Once the expression of the capacity per subchannel is computed, we use equation (4) to derive the optimal transmit power per subchannel:

$$
P_{T_{X}}^{k, n}=B_{c}\left(\frac{2^{\frac{C_{k}}{N_{k}^{\prime} B_{c} \mu_{o p t}}}}{\left(\prod_{n=1}^{N_{k}^{\prime}} G_{k, n}\right)^{\frac{1}{N_{k}^{\prime}}}}-\frac{1}{G_{k, n}}\right)^{\dagger}
$$

We now compute the expression of $\mu_{o p t}$ the optimal service time. With the KKT conditions, each inequality constraint can be either considered as an equality (the constraint is binding) or as a strict inequality (the constraint is non-binding). In the studied problem, the two constraints of (9b) and (9d) can either be binding or non-binding. If the constraint of (9d) is binding, the base station energy consumption is minimized by maximizing the instantaneous transmit power and so minimizing the service time and maximizing sleep mode duration. On the contrary, if the constraint of (9b) is binding the energy consumption is minimized without switching to sleep mode during the frame. Thus, these two constraints can't simultaneously be binding.

As a consequence, if one of the two constraints of (9b) or (9d) is binding, the other will be non-binding. This leads to three different cases:

1) None of these two constraints are binding.

2) Constraint of (9b) is binding $\left(\mu_{a}=1\right)$.

3) Constraint of $(9 d)$ is binding.

We have a different value of $\mu_{o p t}$ for each case.

1) Case 1: In this case, none of the two constraints (9b) and $(9 \mathrm{~d})$ is binding. We denote:

$$
\begin{aligned}
f\left(\mu_{a}\right)=\sum_{k=1}^{N_{u}} \frac{2^{\frac{C_{k}}{N_{k}^{\prime} B_{c} \mu_{a}}}}{\left(\prod_{n=1}^{N_{k}^{\prime}} G_{k, n}\right)^{\frac{1}{N_{k}^{\prime}}}}\left[\frac{C_{k} \ln (2)}{B_{c} \mu_{a}}-N_{k}^{\prime}\right] \\
-\frac{P_{0}-P_{s}}{m B_{c}}+\sum_{k=1}^{N_{u}} \sum_{n=1}^{N_{k}^{\prime}} \frac{1}{G_{k, n}},
\end{aligned}
$$

and $\mu_{o p t}$ is the solution of:

$$
f\left(\mu_{a}\right)=0
$$

The proof is given in appendix B.

It may be noted that in the single-user case, the optimal service time has the following expression:

$$
\begin{aligned}
& \mu_{o p t}=\frac{C \ln (2)}{N^{\prime} B_{c}} \times \\
& \mathcal{W}\left(e^{-1}\left(\prod_{n=1}^{N^{\prime}} G_{n}\right)^{\frac{1}{N^{\prime}}}\left(\frac{P_{0}-P_{s}}{m N^{\prime} B_{c}}-\frac{1}{N^{\prime}} \sum_{n=1}^{N^{\prime}} \frac{1}{G_{n}}\right)\right)+1
\end{aligned}
$$

where $\mathcal{W}$ denotes the W-Lambert function [11] which is the reciprocal bijection of $x \mapsto x e^{x}$. This formula is very similar to the optimal TDMA power allocation [6].
2) Case 2: If (9b) is binding, $\mu_{\text {opt }}=1$. We obtain the power allocation which minimizes the transmit power without sleep mode [12]:

$$
P_{T_{X}}^{k, n}=B_{c}\left(\frac{2^{\frac{C_{k}}{N_{k}^{\prime} B_{c}}}}{\left(\prod_{n=1}^{N_{k}^{\prime}} G_{k, n}\right)^{\frac{1}{N_{k}^{\prime}}}}-\frac{1}{G_{k, n}}\right)^{+}
$$

3) Case 3: If the constraint of (9d) is binding, $\mu_{o p t}$ is the solution of:

$$
B_{c} \sum_{k=1}^{N_{u}}\left(\frac{N_{k}^{\prime} 2^{\frac{C_{k}}{N_{k}^{\prime} B_{c} \mu_{o p t}}}}{\left(\prod_{n=1}^{N_{k}^{\prime}} G_{k, n}\right)^{\frac{1}{N_{k}^{\prime}}}}-\sum_{n=1}^{N_{k}^{\prime}} \frac{1}{G_{k, n}}\right)=P_{\max }
$$

The proof is similar to the one done in case 1 .

In this case, the instantaneous transmit power is maximized when the base station is active, $\mu_{a}$ is minimized and the sleeping time is maximized. In other words, at each iteration of the water-filling algorithm described by equation (11), we know that the RF transmit power can't be higher than a maximum transmit power $P_{\max }$. Consequently, the service time can't be lower than a minimum service time, which is the solution of equation (16). In the following we denote $\mu_{\text {min }}$ this minimum service time.

Finally, to compute the power allocation which minimizes the base station energy consumption, we use the water-filling algorithm described by equation (11). At each iteration, we first compute $\mu_{\min }$ solving equation (16), then we compute $\mu_{\text {opt }}$ solving equation (13). This can be done using single variable root finding algorithms (bisection method, Newton's method, etc.). Then, if $\mu_{o p t}<\mu_{\min }$ (resp. $\mu_{o p t}>1$ ), the service time is set to $\mu_{\min }$ (resp. to 1). Each iteration ends with a computation of the transmit power in subchannels with equation (11).

\section{Resource Allocation}

Once the problem of power allocation is solved, we analyse the problem of resource allocation. Indeed, with $N_{u}$ users and $N_{c}$ subchannels, the number of resource allocations for which each user has at least one subchannel, is equal to [13]:

$$
N_{\text {res }}=\sum_{i=0}^{N_{u}}(-1)^{i}\left(\begin{array}{c}
N_{u} \\
i
\end{array}\right)\left(N_{u}-i\right)^{N_{c}}=\mathcal{O}\left(N_{u}^{N_{c}}\right)
$$

In many cases, $N_{\text {res }}$ is too large to propose an exhaustive search for the optimal resource allocation. That is why in the following, we propose sub-optimal resource allocations.

Equation (11) and (15) give the power allocations which minimize respectively the energy consumption with and without Cell DTx. These two formulas are very similar. That is why, in this section, we use exhaustive searches for a small number of users to compare two strategies. We first analyse the average power consumption with the optimal resource allocation. To do that, we use the algorithm described in the previous section to compute the transmit power with Cell DTx for all the possible resource allocations and we select the one for which the energy consumption is minimized. With 
the second strategy, we use equation (15) to compute the power allocation which minimizes the transmit power for all resource allocations and we choose the resource allocation for which the transmit power is minimized. Then, we use the algorithm described in the previous section to compute the users' transmit power and the service time of the base station with Cell DTx for this resource allocation. In that case, we use a suboptimal resource allocation, the power consumption with this second strategy will be higher than the one obtained with the first strategy. In the following, we numerically evaluate the maximum loss caused by this suboptimal resource allocation.

As in LTE standard, we suppose a band divided in $180 \mathrm{kHz}$ subchannels composed by 12 subcarriers. For each user, we set an average maximum SNR denoted $\rho_{\max }^{i}$ which is the average SNR that would have user $i$ if he was served with all the available transmit power. We use the ETU Extended Typical Urban channel model to generate fading. We make an iterative search over all the possible resource allocations and we analyse the error committed if we use DTx with the optimal resource allocation without DTx.

We first consider scenarios in which the base station serves two users in 6 (1.4 MHz LTE bandwidth) or 15 (3 MHz LTE bandwidth) subchannels. Table II shows the maximum loss obtained for different values of $\rho_{\max }$ and for different base station types. This loss is the ratio between the extra energy consumed by the suboptimal resource allocation and the gain in energy provided by DTx compared to the optimal resource allocation without DTx.

TABLE II

MAXIMUM LOSS WHEN THE OPTIMAL POWER ALLOCATION WITH SLEEP MODE IS USED WITH THE RESOURCE ALLOCATION WHICH MINIMIZES THE TRANSMIT POWER.

\begin{tabular}{|c|c|c|c|c|c|}
\hline BS type & $\rho_{\max }^{1}(\mathrm{~dB})$ & $\rho_{\max }^{2}(\mathrm{~dB})$ & $N_{c}$ & Loss $(\%)$ & Scenario \\
\hline \multirow{4}{*}{ Macro } & \multirow{2}{*}{15} & \multirow{2}{*}{15} & 6 & 1.1 & $\# 1$ \\
\cline { 4 - 6 } & & & 15 & 1.7 & $\# 2$ \\
\cline { 2 - 6 } & \multirow{2}{*}{ Femto } & \multirow{2}{*}{25} & 6 & 10.3 & $\# 3$ \\
\cline { 4 - 6 } & \multirow{2}{*}{15} & \multirow{2}{*}{15} & 15 & 2.5 & $\# 4$ \\
\cline { 2 - 6 } & \multirow{2}{*}{5} & \multirow{2}{*}{25} & 6 & 0.9 & $\# 5$ \\
\cline { 4 - 6 } & & & 15 & 1.3 & $\# 6$ \\
\hline
\end{tabular}

We can see in table II that, in the cases studied, the loss never exceeds $10.5 \%$. Furthermore, we made some simulations with 4 users and 6 subchannels with macrocells and femtocells and the average loss was always below $1 \%$. We show in figure 2 the average power consumption with the optimal and suboptimal strategies in one of the worst case scenarios $\left(7^{\text {th }}\right.$ scenario). We can see that the optimal and suboptimal resource allocations provide similar results.

These numerical evaluations show that for all base station types, there is little interest in developing new algorithms for resource allocations. Indeed, resource allocation algorithms developed for transmit power minimization (power control) will have near optimal performance. Moreover, many solutions have been proposed for transmit power minimization such as

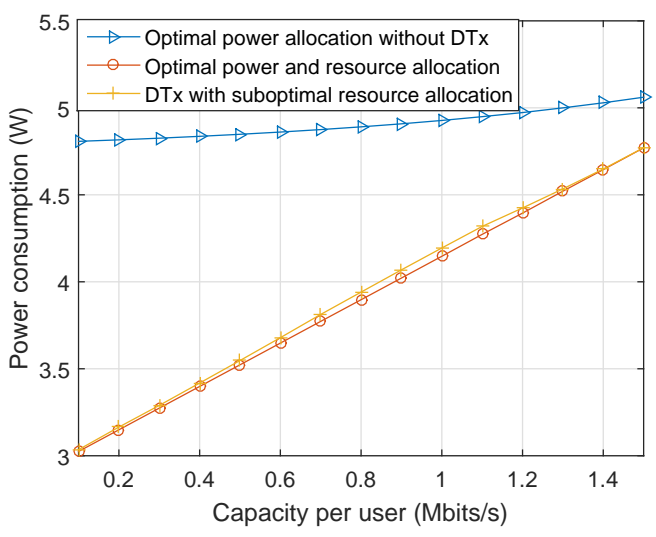

Fig. 2. Average femtocell power consumption in the $7^{\text {th }}$ scenario. We can see that the gap between the optimal resource allocation and the resource allocation which minimizes the transmit power (suboptimal) increases and then decreases as the capacity constraint increases.

in [12], [14]. In this paper, without loss of generality, we use the Bandwidth Assignment Based on SNR (BABS) algorithm to compute the number of subchannels per user and the Amplitude-Craving Greedy (ACG) algorithm for subchannel assignment. Both algorithms have been proposed in [9]. The main advantage of the BABS+ACG algorithm is its low complexity which is $O\left(N_{u} N_{c}\right)$.

\section{Numerical Results}

In a first step, we consider some of the scenarios introduced in the previous section. We suppose that a base station serves two users with a $1.4 \mathrm{MHz}$ band divided in 6 subchannels of 12 subcarriers. We compare the base station average power consumption with the optimal resource allocation and with the $\mathrm{BABS}+\mathrm{ACG}$ algorithm. All the results are displayed in figure 3. For low capacity constraint, the BABS+ACG algorithm is near optimal and DTx saves up to $37.5 \%$ of a femtocell energy consumption and $38.5 \%$ of a macrocell energy consumption. Furthermore, we can see in figure $3 \mathrm{a}$ and $3 \mathrm{c}$ that when the two users have the same SNR, the BABS+ACG resource allocation is optimal with and without DTx. Furthermore, when one user has a low SNR and the other a high SNR, the energy consumption with the BABS+ACG resource allocation moves away from the optimal average power consumption as the capacity constraint increases. We can see in figure $3 \mathrm{~b}$ that for a macrocell, the gap between the optimal power consumption and the power consumption with the BABS+ACG algorithm is the same with and without DTx. In this case, this gap is only caused by the resource allocation and can be reduced with more efficient resource allocations (with a higher complexity). On the contrary, we observe in figure $3 \mathrm{~d}$ that when the BABS+ACG is used for femtocells with Cell DTx, There is a gap caused by the suboptimality of the resource allocation which minimizes the transmit power. The loss observed in figure 2 is amplified by the suboptimality of the BABS+ACG algorithm. However, the dynamic part of the power consumption is less important for femtocells. Consequently, we have approximately the same loss caused by the suboptimal resource allocation for femtocells (5\% of 


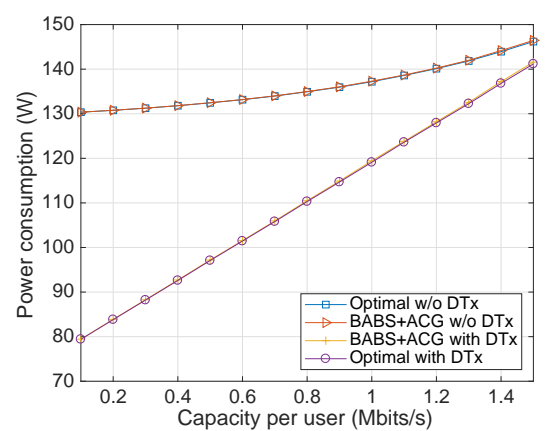

(a) Macrocell, $\rho_{\text {max }}^{1}=\rho_{\text {max }}^{2}=15 d B$

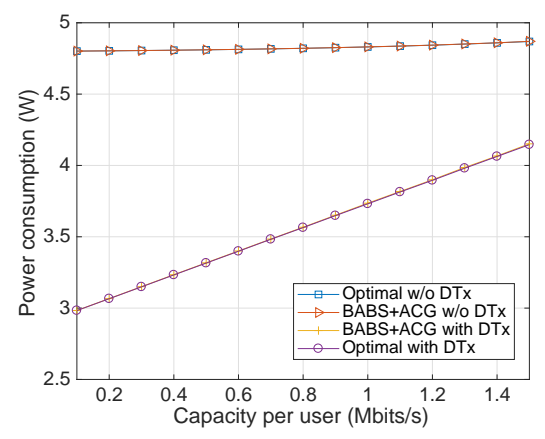

(c) Femtocell, $\rho_{\text {max }}^{1}=\rho_{\text {max }}^{2}=15 d B$

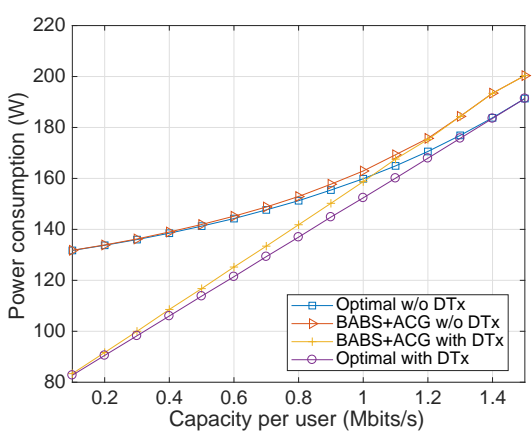

(b) Macrocell, $\rho_{\text {max }}^{1}=5 \mathrm{~dB}, \rho_{\text {max }}^{2}=25 \mathrm{~dB}$

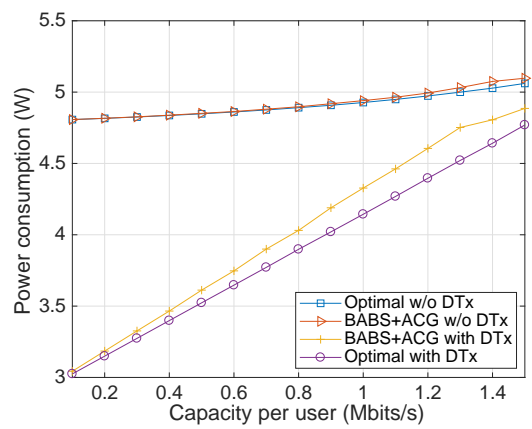

(d) Femtocell, $\rho_{\max }^{1}=5 d B, \rho_{\max }^{2}=25 d B$

Fig. 3. Comparison of the proposed approach with the optimal solution when 2 users are served in 6 subchannels (1.4MHz band).

the total power consumption) and for macrocells (6\% of the total power consumption).

We now study the performance of Cell DTx in a realistic scenario. We suppose that a macrocell serves some users distributed according to a Poisson Point Process (PPP) in a $20 \mathrm{MHz}$ band divided in 100 subchannels. The central frequency is $2 \mathrm{GHz}$. Moreover, the average number of users in the cell coverage is equal to 10 and the distance between the users and the base station varies between 300 and $1500 \mathrm{~m}$. The pathloss is computed with the Winner II suburban macrocell pathloss model. The antenna gain is set to $10 \mathrm{dBi}$. The ETU Extended Pedestrian A model is used to generate fading and a $2 \mathrm{~dB}$ noise figure is added. We compare the optimal power allocations with the BABS+ACG algorithm with and without Cell DTx.

We can see the evolution of the average power consumption with and without DTx in figure 4. With the BABS+ACG algorithm, DTx saves up to $38 \%$ of the base station energy consumption. The energy saved with DTx decreases as the capacity constraint increases. Indeed, when the capacity constraint increases, the dynamic part of the power consumption (which is proportional to the transmit power) increases and the optimal strategy becomes closer to the optimum without DTx. For a capacity constraint of 8 Mbits/s DTx saves $2 \%$ of the base station energy consumption.

\section{CONCLUSION}

In this paper, we study the problem of power and resource allocation in OFDMA with Cell DTx. We prove that the problem of power allocation can be rewritten as a convex problem and can be solved using a water-filling algorithm and single variable root-finding algorithms. Moreover, we show that the algorithms developed for power control without DTx have good performance when DTx is used. We use the BABS+ACG algorithm and we show that DTx can save up to $38 \%$ of base station energy consumption. In our future work, we will study the problem of power allocation with Cell DTx in multicell scenarios.

\section{ACKNOWLEDGEMENT}

Part of this work is supported by the project SOGREEN, which is funded by the French national research agency, under the grant agreement coded: N ANR-14-CE28-0025-02 and by Région Bretagne, France.

\section{APPENDIX A}

\section{NON-CONVEXITY OF THE FIRST PROBLEM}

To prove the non-convexity of the problem introduced in equation (3), we can either prove that the objective function of equation (3a) or the constraints of equation (3c) are not convex as function of $\mu_{a}$ and the transmit powers $P_{T_{X}}^{k, n}$ for all $k, n$. The proof is made for the constraints of equation (3c). We denote:

$$
\begin{gathered}
\alpha_{n}=\frac{1}{\ln (2)} \frac{G_{k, n}}{1+\frac{G_{k, n} P_{T_{X}}^{k, n}}{B_{c}}} \geq 0 \\
\beta_{n}=\frac{G_{k, n}^{2} \mu_{a}}{B_{c} \ln (2)} \frac{1}{\left(1+\frac{G_{k, n} P_{T_{X}}^{k, n}}{B_{c}}\right)^{2}} \geq 0
\end{gathered}
$$




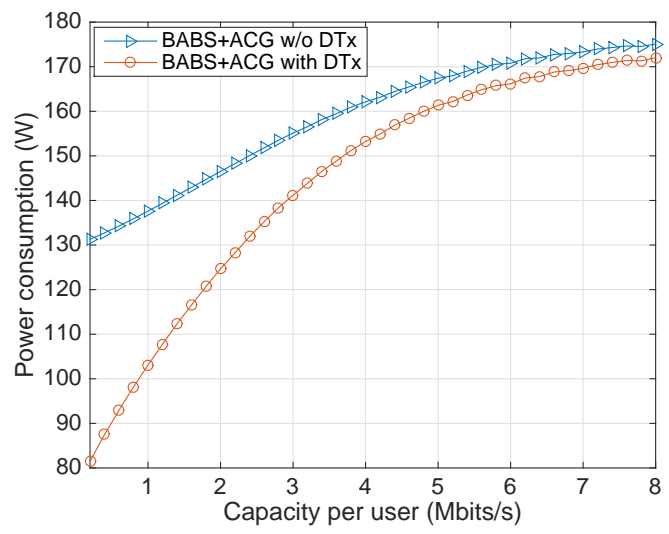

Fig. 4. Average base station power consumption with and without DTx with the BABS+ACG algorithm. DTx allows to save up to $38 \%$ of the base station energy consumption.

For $k \in \llbracket 1 ; N_{u} \rrbracket$, the calculation of the Hessian matrix of (3c) gives:

$$
H_{k}=\left(\begin{array}{cccc}
0 & -\alpha_{1} & \cdots & -\alpha_{N_{k}} \\
-\alpha_{1} & \beta_{1} & \cdots & 0 \\
\vdots & \vdots & \ddots & \vdots \\
-\alpha_{N_{k}} & 0 & \cdots & \beta_{N_{k}}
\end{array}\right)
$$

$\operatorname{Tr}\left(H_{k}\right)>0$, thus, at least one eigenvalue of $H_{k}$ is strictly positive. Expanding along the first row, we can compute the determinant of this matrix:

$$
\operatorname{det}\left(H_{k}\right)=-\sum_{n=1}^{N_{k}} \alpha_{n}^{2} \prod_{j=1, j \neq n}^{N_{k}} \beta_{j}<0
$$

Thus the product of the eigenvalues of $H_{k}$ is negative and $H_{k}$ has at least one strictly negative eigenvalue. $H_{k}$ has at least a strictly negative and a strictly positive eigenvalue. As a consequence, the constraint $(3 \mathrm{c})$ is not convex.

\section{APPENDIX B}

\section{OPTIMAL SERVICE TIME AND POWER ALLOCATION}

The proof is done for the first case. The Lagrangian of the problem studied is:

$$
\begin{array}{r}
\mathcal{L}=P_{s}+\mu_{a}\left(P_{0}-P_{s}\right)+m \mu_{a} B_{c} \sum_{k=1}^{N_{u}} \sum_{n=1}^{N_{k}} \frac{1}{G_{k, n}}\left(2^{\frac{C_{k, n}}{B_{c} \mu_{a}}}-1\right) \\
-\sum_{k=1}^{N_{u}} \lambda_{k}\left(\sum_{n=1}^{N_{k}} C_{k, n}-C_{k}\right)
\end{array}
$$

The derivative of the Lagrangian with respect to $C_{k, n}$ is

$$
\frac{\partial \mathcal{L}}{\partial C_{k, n}}=\frac{m \ln (2)}{G_{k, n}} 2^{\frac{C_{k, n}}{B_{c} \mu_{a}}}-\lambda_{k}
$$

At the minimum, this derivative is zero, thus:

$$
C_{k, n}=B_{c} \mu_{a} \log _{2}\left(\frac{\lambda_{k} G_{k, n}}{m \ln (2)}\right)
$$

Since $\sum_{n=1}^{N_{k}} C_{k, n}=C_{k}$, we have:

$$
B_{c} \mu_{a} \log _{2}\left(\frac{\lambda_{k}}{m \ln (2)}\right)=\frac{C_{k}}{N_{k}}-\frac{B_{c} \mu_{a}}{N_{k}} \log _{2}\left(\prod_{n=1}^{N_{k}} G_{k, n}\right)
$$

Thus,

$$
C_{k, n}=\frac{C_{k}}{N_{k}}+B_{c} \mu_{a} \log _{2}\left(\frac{G_{k, n}}{\left(\prod_{n=1}^{N_{k}} G_{k, n}\right)^{\frac{1}{N_{k}}}}\right)
$$

Furthermore, the differential of the Lagrangian with respect to $\mu_{a}$ is:

$$
\begin{array}{r}
\frac{\partial \mathcal{L}}{\partial \mu_{a}}=\sum_{k=1}^{N_{u}} \sum_{n=1}^{N_{k}}\left[\frac{m B_{c}}{G_{k, n}}\left(2^{\frac{C_{k, n}}{B_{c} \mu_{a}}}-1\right)-\frac{m C_{k, n} \ln (2)}{G_{k, n} \mu_{a}} 2^{\frac{C_{k, n}}{B_{c} \mu_{a}}}\right] \\
+\left(P_{0}-P_{s}\right)
\end{array}
$$

By replacing $C_{k, n}$ in (27) by its expression (26), $\frac{\partial \mathcal{L}}{\partial \mu_{a}}=0$ gives:

$$
\begin{aligned}
\sum_{k=1}^{N_{u}} \frac{2^{\frac{C_{k}}{N_{k} B_{c} \mu_{a}}}}{\left(\prod_{n=1}^{N_{k}} G_{k, n}\right)^{\frac{1}{N_{k}}}}\left[\frac{C_{k} \ln (2)}{B_{c} \mu_{a}}-N_{k}\right] \\
-\frac{P_{0}-P_{s}}{m B_{c}}+\sum_{k=1}^{N_{u}} \sum_{n=1}^{N_{k}} \frac{1}{G_{k, n}}=0
\end{aligned}
$$

Then, by successively setting to zero all the capacities below zero, according to water-filling principle, we obtain the optimal capacity per subchannel. We can finally compute the optimal power allocation per subchannel.

\section{REFERENCES}

[1] A. Fehske and al., "The global footprint of mobile communications: The ecological and economic perspective," Communications Magazine, IEEE, vol. 49, pp. 55-62, August 2011.

[2] P. Frenger and al., "Reducing Energy Consumption in LTE with Cell DTX," in Vehicular Technology Conference (VTC Spring), 2011 IEEE 73rd, pp. 1-5, May 2011.

[3] H. Holtkamp, G. Auer, and H. Haas, "On Minimizing Base Station Power Consumption," in Vehicular Technology Conference (VTC Fall), 2011 IEEE, pp. 1-5, Sept 2011.

[4] R. Wang, J. S. Thompson, H. Haas, and P. M. Grant, "Sleep mode design for green base stations," IET Communications, vol. 5, pp. 2606-2616, Dec 2011.

[5] R. Bonnefoi, C. Moy, and J. Palicot, "Dynamic Sleep Mode for Minimizing a Femtocell Power Consumption," in CROWNCOM, May 2016.

[6] R. Bonnefoi, C. Moy, and J. Palicot, "New Macrocell Downlink Energy Consumption Minimization with Cell DTx and Power Control," in IEEE International Conference on Communications, pp. 1-7, May 2017.

[7] H. Holtkamp, G. Auer, S. Bazzi, and H. Haas, "Minimizing Base Station Power Consumption," IEEE Journal on Selected Areas in Communications, vol. 32, pp. 297-306, February 2014.

[8] H. B. Ren, M. Zhao, J. K. Zhu, and W. Y. Zhou, "Energy-efficient resource allocation for OFDMA networks with sleep mode," Electronics Letters, vol. 49, pp. 111-113, January 2013.

[9] D. Kivanc, G. Li, and H. Liu, "Computationally efficient bandwidth allocation and power control for OFDMA," IEEE Transactions on Wireless Communications, vol. 2, pp. 1150-1158, Nov 2003.

[10] G. Auer and al., "How much energy is needed to run a wireless network?," IEEE Wireless Commun., vol. 18, pp. 40-49, October 2011.

[11] R. Corless and al., "On the LambertW function," Advances in Computational Mathematics, vol. 5, no. 1, pp. 329-359, 1996.

[12] K. Seong, M. Mohseni, and J. M. Cioffi, "Optimal Resource Allocation for OFDMA Downlink Systems," in 2006 IEEE International Symposium on Information Theory, pp. 1394-1398, July 2006.

[13] N. Loehr, Bijective Combinatorics. Chapman \& Hall/CRC, 1st ed., 2011.

[14] C. Y. Wong, R. S. Cheng, K. B. Lataief, and R. D. Murch, "Multiuser OFDM with adaptive subcarrier, bit, and power allocation," IEEE Journal on Selected Areas in Communications, vol. 17, pp. 1747-1758, Oct 1999. 